\title{
Optimized threshold calculation for blanking nonlinearity at OFDM receivers based on impulsive noise estimation
}

\author{
Zehra Ali ${ }^{1}$, Ferheen Ayaz ${ }^{2^{*}}$ and Chang-Soo Park ${ }^{1 *}$
}

\begin{abstract}
Impulsive noise (IN) degrades the performance of OFDM-based communication systems. Performance degradation is usually measured in terms of signal-to-noise ratio (SNR) and symbol-error rate (SER). To improve the performance of the system of OFDM receivers, one of the effective methods is to use blanking nonlinearity. In this method, the samples whose magnitudes exceed a certain fixed threshold are considered to be IN-affected and are therefore blanked. A fixed threshold does not always determine the IN-affected samples truly for all probabilities of IN occurrence. This paper proposes an optimized threshold-calculation method for blanking nonlinearity which is based on distribution characteristics of the received signal, i.e., mean, median, and peak. The proposed method can calculate an optimized threshold for all probabilities of impulsive noise occurrence. Simulation results show over 2.2-dB gain in SNR and lower SER by using the proposed method as compared to fixed threshold.
\end{abstract}

Keywords: OFDM; Blanking nonlinearity; Impulsive noise

\section{Introduction}

Impulsive noise (IN) is one of the most challenging factors of performance degradation in power line communications [1]. One of the most widely used modulation techniques for PLC and wireless/wired channels is orthogonal frequency division multiplexing (OFDM). It offers many advantages like increased data rates, robustness in multipath, and resistance to noise [2].

Impulsive noise (IN) is characterized by random occurrences of short bursts with high amplitude [3]. Highspectral-power IN pulses degrade the performance of the OFDM system by reducing its signal-to-noise ratio (SNR) and increasing symbol-error rate (SER) [4]. One of the simple methods to improve system performance is to precede a conventional OFDM demodulator with blanking nonlinearity in which a signal sample is set to zero if its amplitude exceeds a particular threshold. This threshold is conventionally fixed in an OFDM receiver [5]. The fixed value of threshold plays a very important

\footnotetext{
*Correspondence: ferheen@msn.com; csp@gist.ac.kr

${ }^{2}$ Department of Electronic Engineering, NED University of Engineering and Technology, Karachi, Pakistan

${ }^{1}$ School of Information and Communication, Gwangju Institute of Science and Technology (GIST), Gwangju, South Korea
}

role in the resulting SNR of the system. This is because the samples whose amplitudes are greater than the threshold are presumed to be affected by IN and are therefore blanked. Samples whose amplitudes are lesser than the fixed threshold are considered to be true values of the signal-unaffected by IN and hence remain unchanged. Therefore, fixing a very high value of threshold may not blank all the IN-affected signals, and a very low value of threshold may blank the true samples [6]. Figures 1 and 2 explain the significant role of threshold.

Various methods to find optimal threshold have been reported in [7] and [8]. However, these methods are purely theoretical. A direct relationship between the peak of the transmitted signal and optimal threshold is derived in [8], but it is not feasible to determine the exact peak of transmitted signal of OFDM receivers. In this paper, we propose an optimized threshold-calculation method for blanking nonlinearity based on impulsive noise estimation (INE) by using distribution characteristics of the received signal, i.e., peak (maximum value), median, and mean. The use of median and mean in filtering and removing impulsive noise has already resulted in many wellestablished algorithms in [9] and [10]. The optimized threshold-calculation method proposed in this paper is 


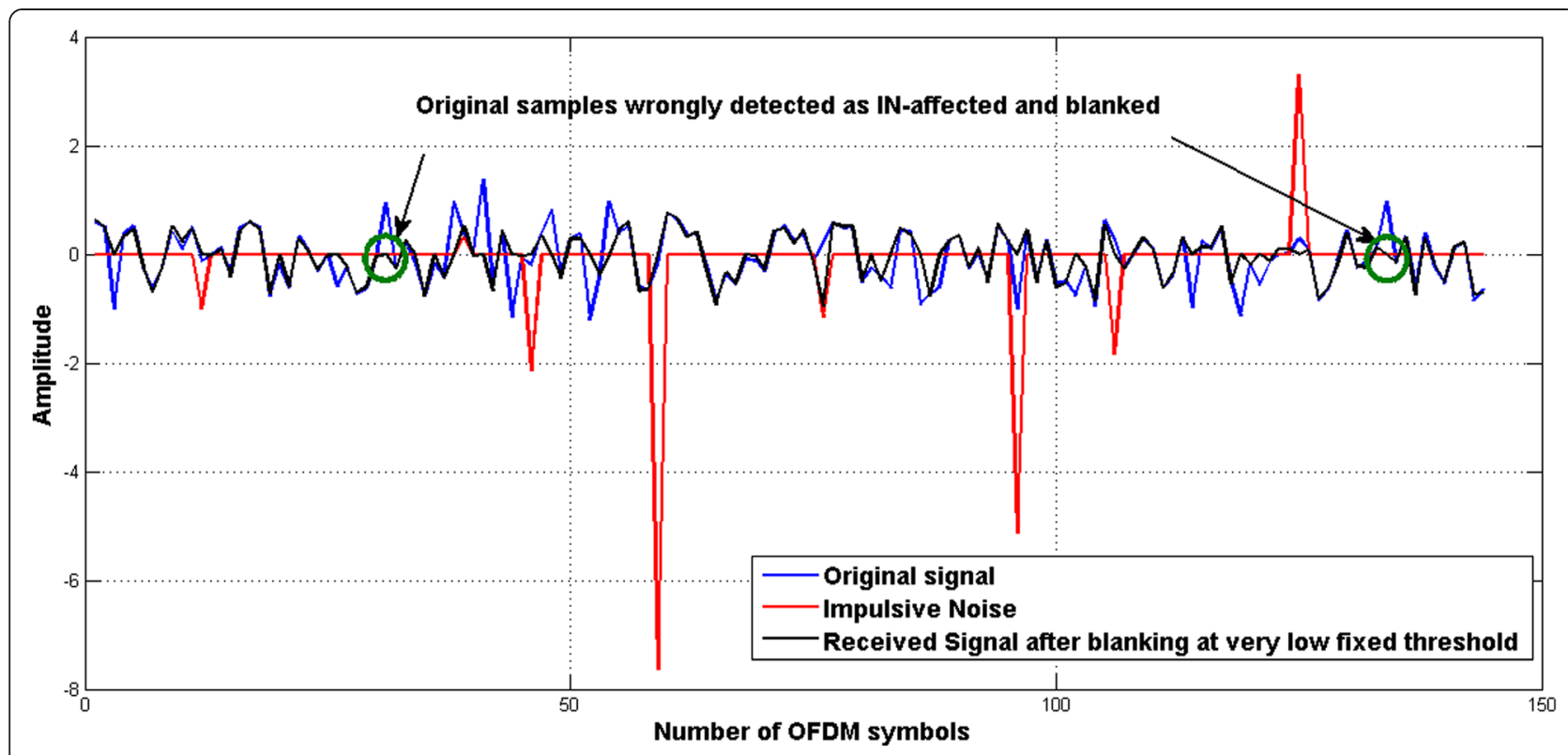

Fig. 1 Blanking nonlinearity at very low fixed threshold applied to IN-affected signal

independent of the probability of IN occurrence and can be adapted to a receiver even if the probability of IN occurrence in the channel is not known. Since the proposed method determines the optimized threshold using the characteristics of the received signal, instead of the transmitted signal, it can be used in practical scenarios.

The paper is organized as follows. Section OFDM system model and optimized threshold-calculation method describes the system model and optimized thresholdcalculation method. The system performance of the proposed optimized threshold (OT) is compared with a fixed threshold (FT) by simulations at different probabilities in Section Simulation results and discussions. Conclusions are presented in Section Conclusions.

\section{OFDM System model and optimized threshold- calculation method \\ 2.1 OFDM system model}

The model for OFDM transmission system is shown in Fig. 3.

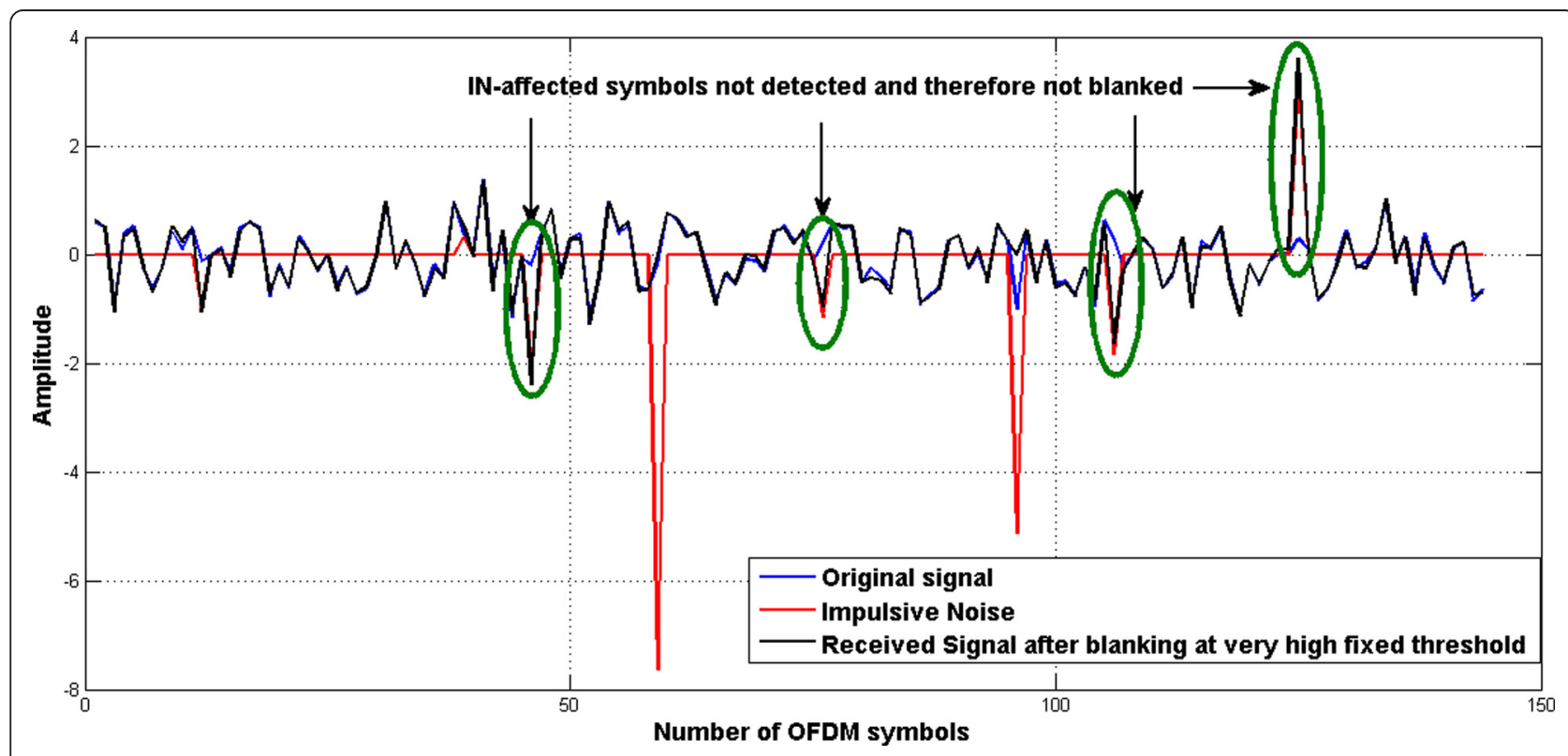

Fig. 2 Blanking nonlinearity at very high fixed threshold applied to IN-affected signals 


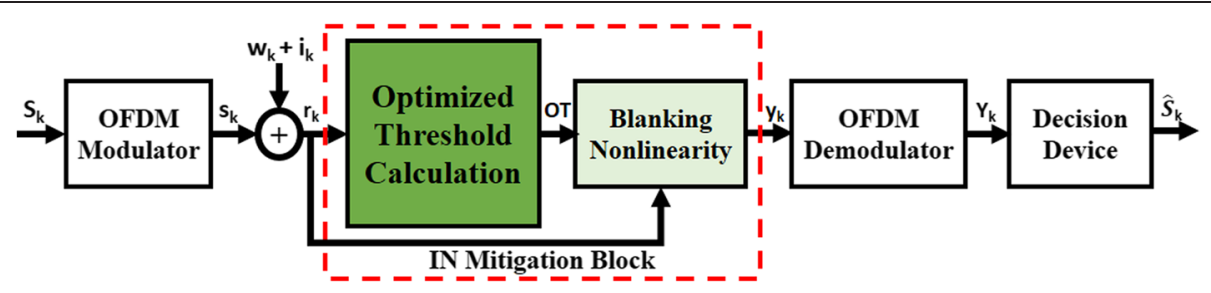

Fig. 3 Block diagram of the OFDM transmission system with the blanking device at the receiver

In the OFDM system, the time-domain signal $s(t)$ is obtained by taking the inverse Fourier transform of the frequency-domain signal by using

$$
s(t)=\frac{1}{\sqrt{N}} \sum_{k=0}^{N-1} S_{k} \exp \left(j \frac{2 \pi \cdot k \cdot t}{T_{S}}\right), \quad 0<t<T_{\mathrm{S}}
$$

Where $S_{k}$ denotes the frequency-domain signal, $j=\sqrt{-1}$, $N$ is the number of subcarriers, and $T_{s}$ is the active symbol interval. The transmitted signal is passed through an additive transmission channel, including additive white Gaussian noise (AWGN) and additive IN. AWGN is denoted by $w_{k}$ and has variance. $\sigma_{w}^{2}=(1 / 2) E\left[\left|w_{k}\right|^{2}\right]$. IN is denoted by $i_{k}$ and is modeled as a Bernoulli-Gaussian random process [4] as follows:

$$
i_{k}=b_{k} g_{k}, \quad 0 \leq k \leq N-1
$$

Where $b_{k}$ is the Bernoulli process of sequence ' $b_{k}=1$ ' or ' $b_{k}=0$ ' with probability of $p$ or $1-p$, respectively, and $g_{k}$ is the complex-zero mean white Gaussian noise with variance $\sigma_{i}^{2}=(1 / 2) E\left[\left|g_{k}\right|^{2}\right]$. Thus, the noisy channel can be characterized by the signal-to-AWGN ratio $S$ $N R=10 \log _{10}\left(1 / \sigma_{w}^{2}\right)$ and signal-to-IN ratio $\operatorname{SINR}=10$ $\log _{10}\left(1 / \sigma_{i}^{2}\right)$. The received time-domain signal $\left(r_{k}\right)$ can be expressed as

$$
r_{k}=\left\{\begin{array}{lc}
s_{k}+w_{k} & \text { if } b_{k}=0 \\
s_{k}+w_{k}+i_{k} & \text { if } b_{k}=1
\end{array}\right.
$$

Where $s_{k}=s\left(k T_{s} / N\right)$ is the sampled OFDM signal in time domain with a variance of $\sigma_{s}^{2}=(1 / 2) E\left|s_{k}\right|^{2}=1$. The received signal is passed to the nonlinear preprocessor. The output of the nonlinear preprocessor $y_{k}$ is then fed to the OFDM demodulator for further processing and is represented in [5] as

$$
\text { Blanking : } y_{k}=\left\{\begin{array}{l}
r_{k}\left|r_{k}\right| \leq T \\
0\left|r_{k}\right|>T
\end{array}\right.
$$

where $T$ is the blanking threshold and $k=0,1, \ldots, N-$ 1. The threshold value is a crucial parameter in blanking

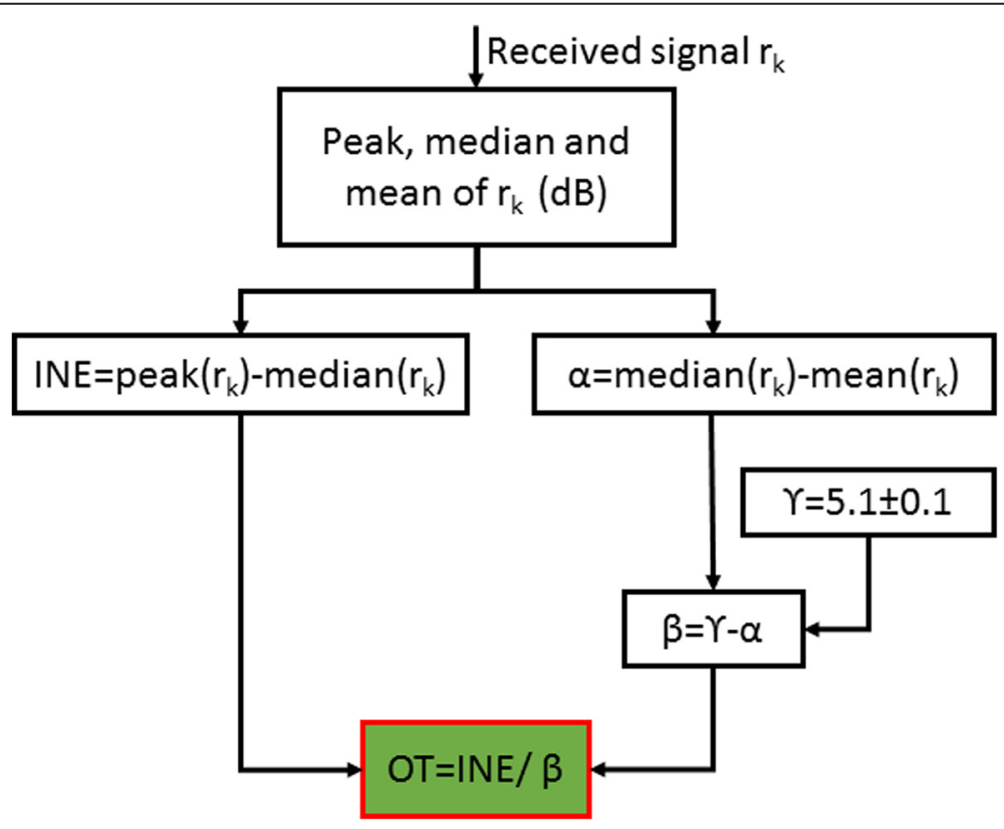

Fig. 4 Block diagram of calculating optimized threshold from distribution characteristics of received signals 


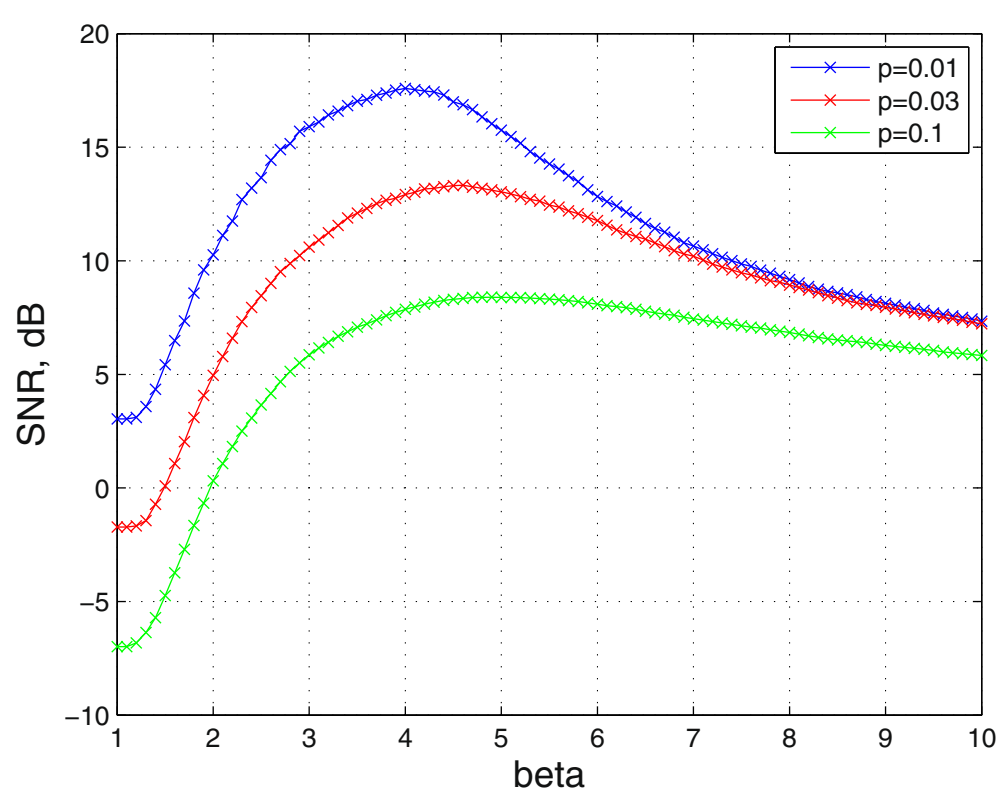

Fig. 5 SNR with varying $\beta$ in OT

nonlinearity as it is responsible for deciding which signal is to be blanked [6].

\subsection{The proposed optimized threshold-calculation} method

We investigated that instead of fixing a threshold, blanking can result in higher SNR if it is performed at an optimized threshold (OT) determined by the distribution characteristics of the received signal. The proposed calculation method for OT is shown in the form of a block diagram in Fig. 4.

For optimizing threshold, we define some parameters known as impulsive noise estimation (INE), alpha $(\alpha)$, beta $(\beta)$, and gamma $(\gamma)$. The value of $\gamma$ can be set by the user in the range of $5.1 \pm 0.1$. The values of INE, $\alpha$, and $\beta$ are calculated as shown in Eqs. (5), (6), and (7) as follows:

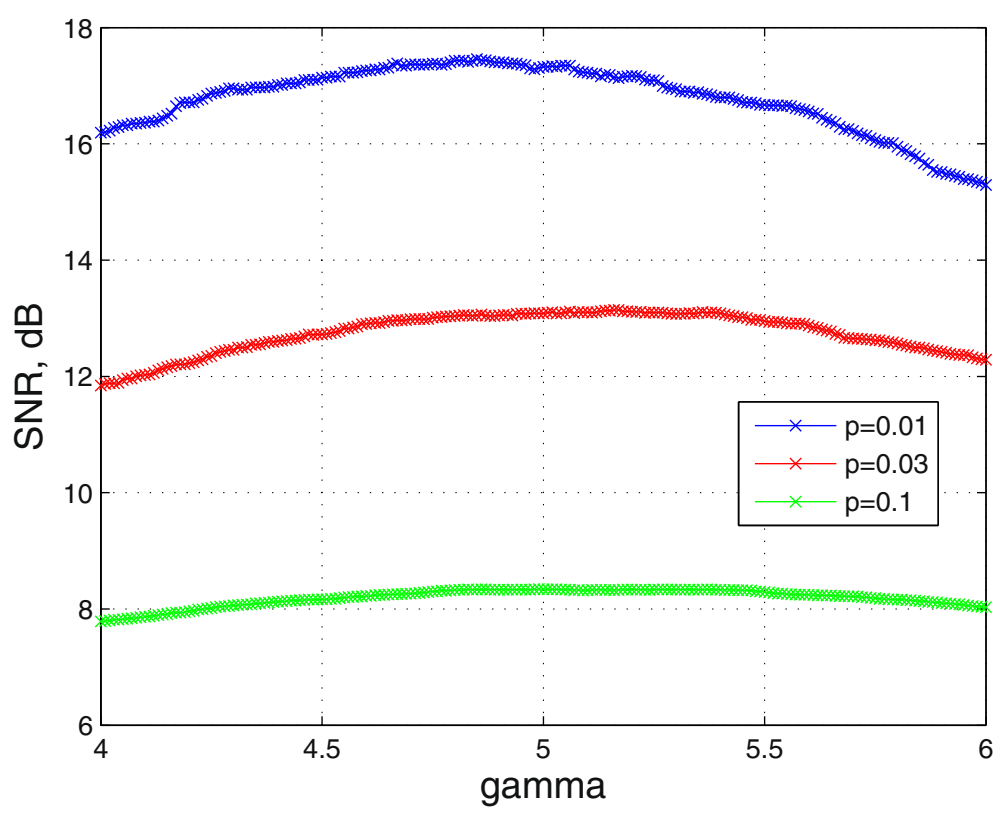

Fig. 6 SNR with varying $\gamma$ in OT 


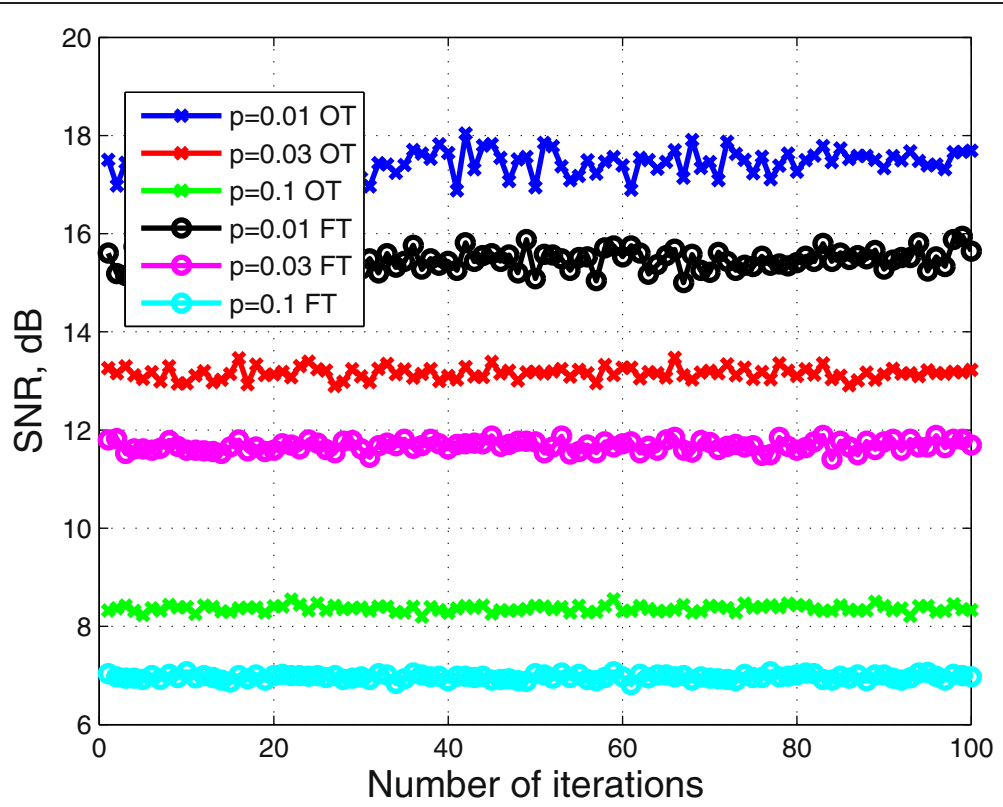

Fig. 7 SNR of blanking nonlinearity with FT and OT for 100 iterations and $p=0.1, p=0.03$, and $p=0.01$

$$
\begin{aligned}
& \mathrm{INE}=\max \left(r_{k}(d B)\right)-\operatorname{mean}\left(r_{k}(d B)\right) \\
& \alpha=\operatorname{median}\left(r_{k}(d B)\right)-\operatorname{mean}\left(r_{k}(d B)\right) \\
& \beta=\gamma-\alpha
\end{aligned}
$$$$
\mathrm{OT}=\mathrm{INE} / \beta
$$

The threshold which gives maximum SNR based on INE is calculated as shown in Eq. (8).
(6)

\section{Simulation results and discussions}

The computer simulation of the OFDM system is carried out with 16 QAM modulation, SNR $=40 \mathrm{~dB}, \mathrm{SINR}=-15$ $\mathrm{dB}$, number of subcarriers, $N=10^{5}, \sigma_{w}^{2}=(1 / 2) E\left[\left|w_{k}\right|^{2}\right]$, and $\sigma_{i}^{2}=(1 / 2) E\left[\left|g_{k}\right|^{2}\right]$.

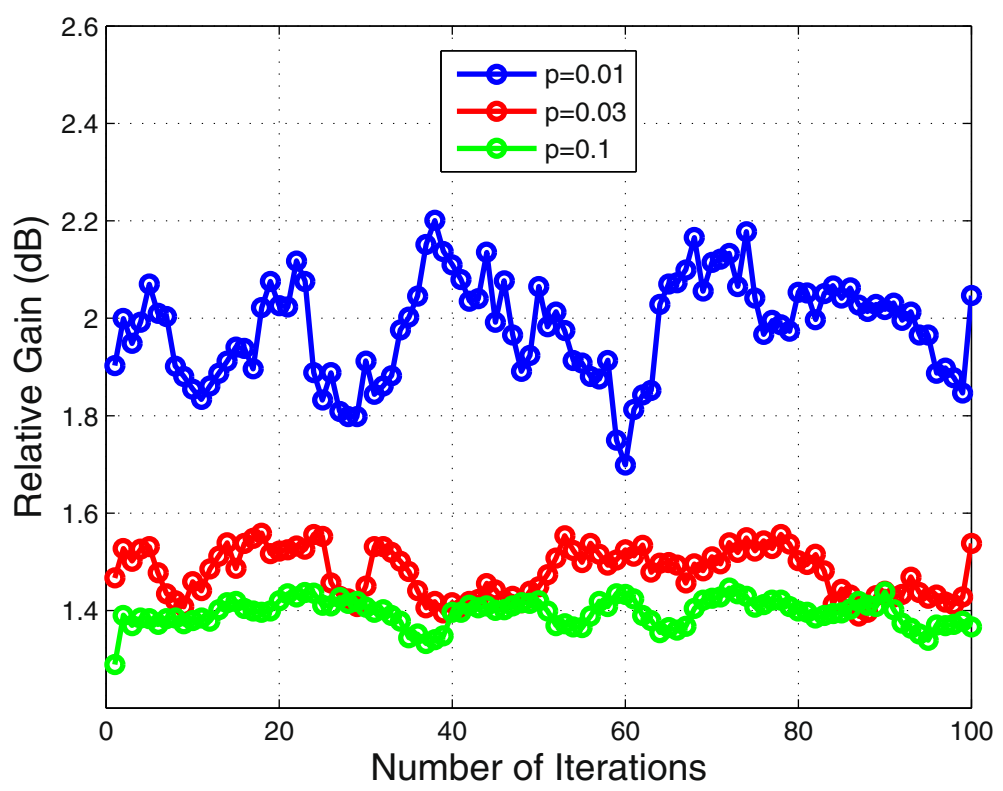

Fig. 8 Relative SNR gain with OT relative to FT blanking nonlinearity, 16 QAM modulation 


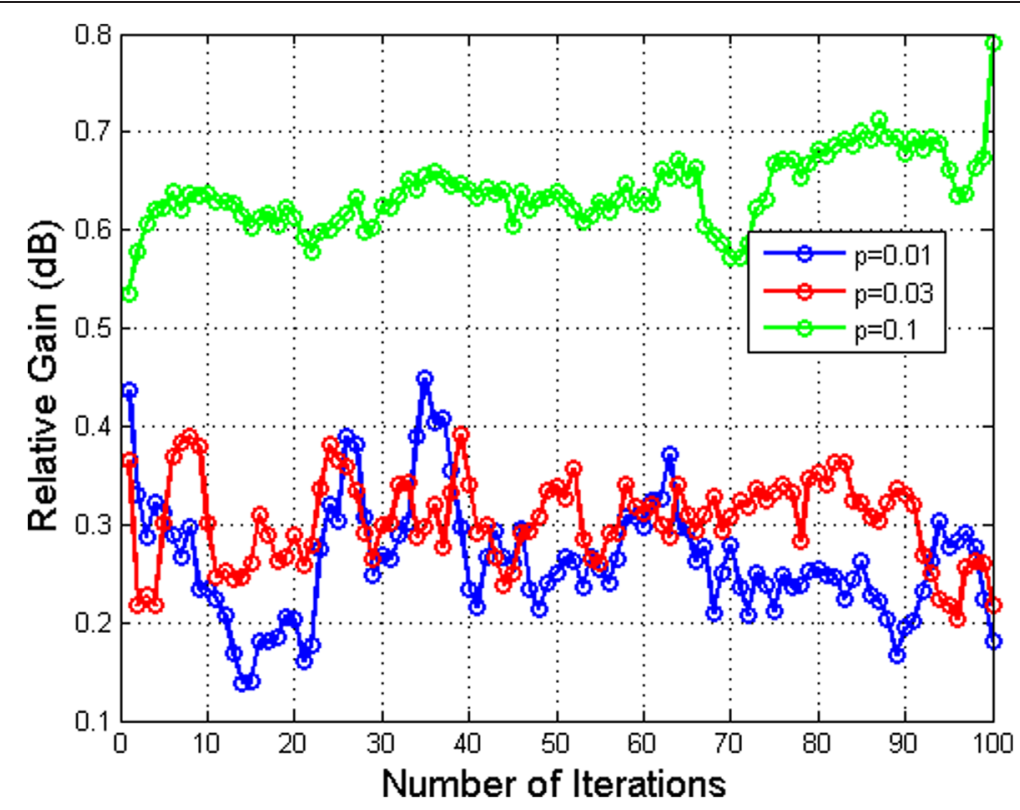

Fig. 9 Relative SNR gain with OT relative to FT blanking nonlinearity, 64 QAM modulation

Probability $(p)$ of IN occurrence is considered to be $p=$ $0.01,0.03$, and 0.1 , which implies that 1,3 , and $10 \%$ of the received samples will be affected by $\mathrm{IN}$, respectively. Therefore, with $10^{5}$ subcarriers, the average number of IN pulses received within each OFDM symbol is $(\mathrm{pN})$, i.e., about 1000, 3000, and 10,000 IN pulses per OFDM symbol for $p=0.01,0.03$, and $p=0.1$, respectively. The output SNR is obtained by

$$
S N R=\left(\frac{E\left[\left|s_{k}\right|^{2}\right]}{E\left[\left|y_{k}-s_{k}\right|^{2}\right]}\right)
$$

Conventionally, the fixed threshold (FT) of OFDM receivers may be selected in the range from 0 to 15 resulting in an SNR of 0 to $18 \mathrm{~dB}$ [5]. It is also found that the value of optimal threshold which gives maximum SNR is

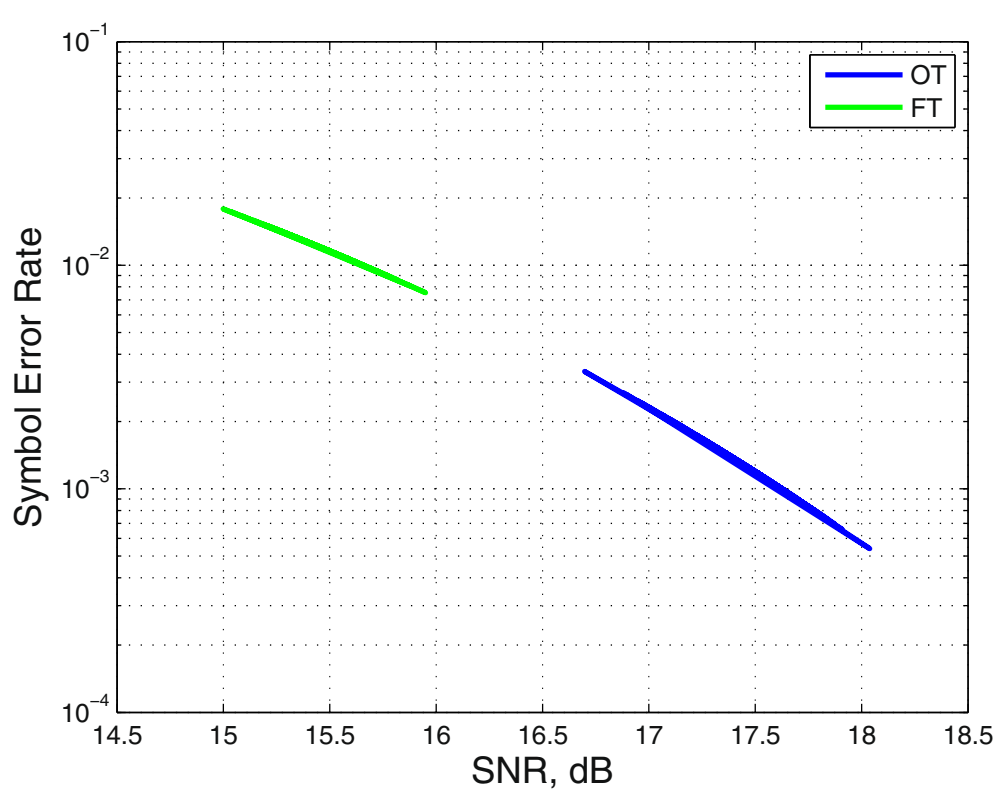

Fig. 10 SER of blanking nonlinearity with OT and FT $p=0.01$ 


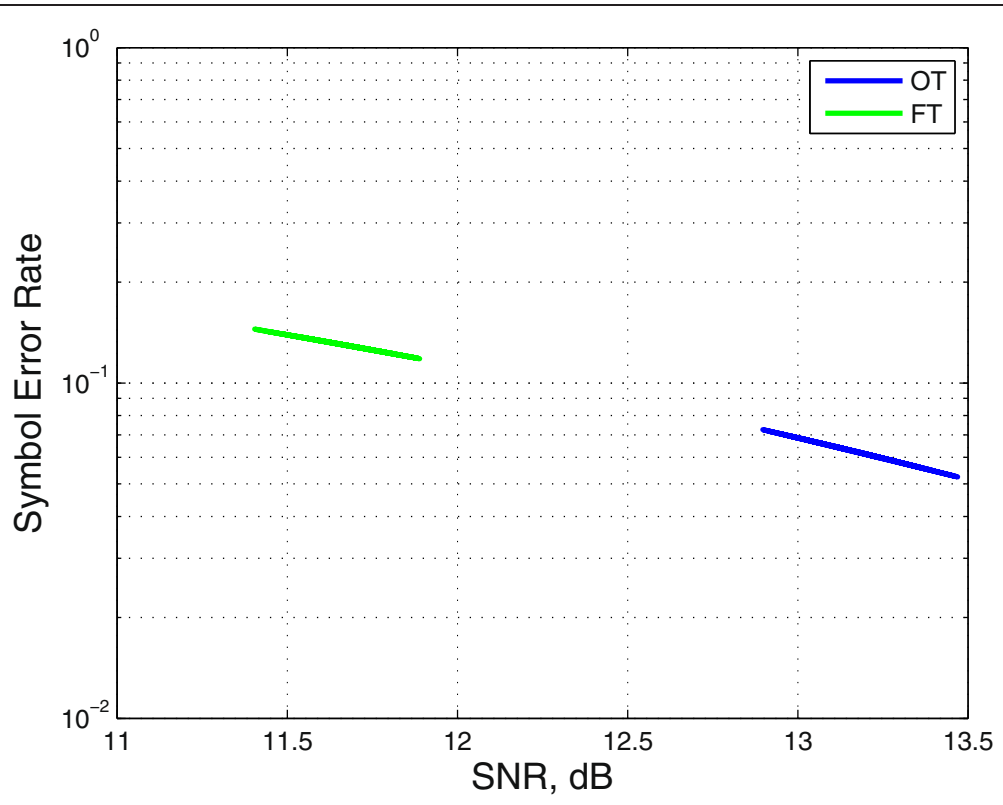

Fig. 11 SER of blanking nonlinearity with OT and FT for $p=0.03$

different for different probabilities of impulsive noise occurrence [6]. In our method of calculating optimized threshold (OT) in Eq. (8), optimum value of $\beta$ lies in the range of 4 to 6 as shown in Fig. 5. In order to get more accurate results, we define $\beta$ as in Eq. (7). Therefore, we have only one fixed parameter, gamma $(\gamma)$, whose value can be $5.1 \pm 0.1$. As shown in Fig. 6, SNR fluctuates only \pm 0.15 $\mathrm{dB}$ by changing the value of $\gamma$ within \pm 0.1 range.

For blanking with FT, the fixed optimal thresholds which maximize SNR were obtained from the results reported in [6]. To check the accuracy of our simulation model in terms of the selection of FT, the SNR after blanking nonlinearity using FT was obtained in 100 iterations. Figure 7 shows that the simulation results of SNR with FT closely match the analytical results as obtained in [6]. Figure 7 also shows the plot of resulting SNR in 100 iterations after applying blanking nonlinearity using OT. It is clearly shown that OT outperforms FT in all IN scenarios by providing a higher SNR than that achieved by FT.

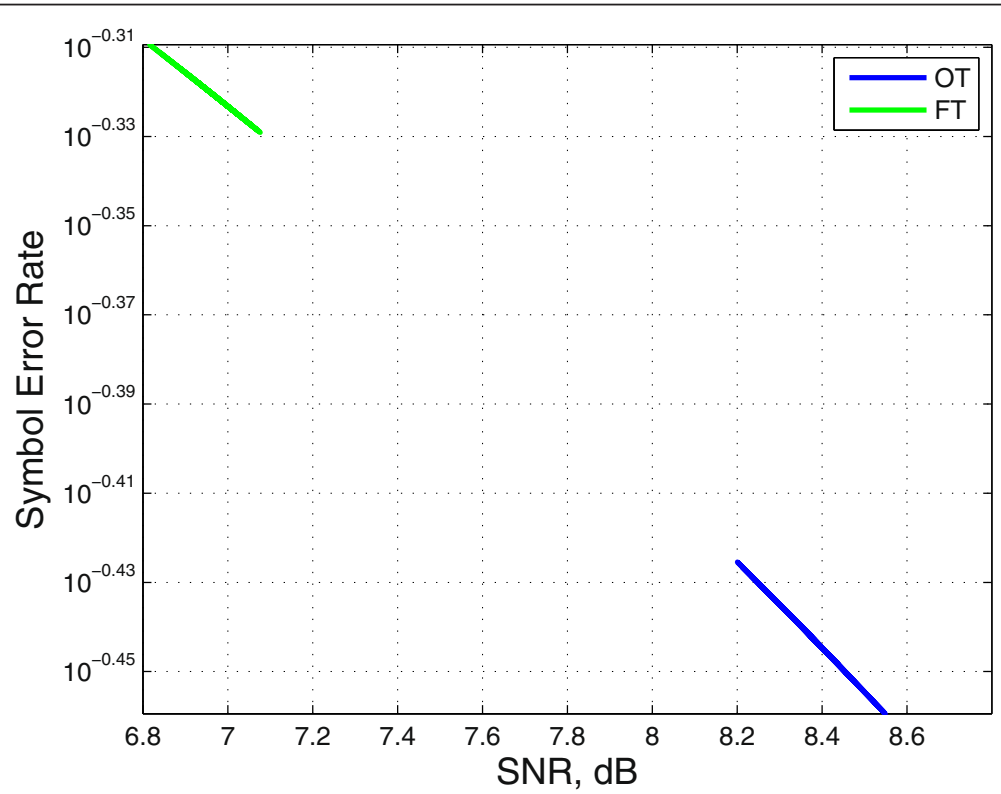

Fig. 12 SER with OT and FT for $p=0.1$ 
Table 1 Minimum SER achieved by blanking nonlinearity with OT and FT

\begin{tabular}{lll}
\hline$p$ & OT & FT \\
\hline 0.01 & $5.6537^{*} 10^{-4}$ & $7.0^{*} 10^{-3}$ \\
0.03 & $5.53^{*} 10^{-2}$ & $9.11^{*} 10^{-2}$ \\
0.1 & $3.49^{*} 10^{-1}$ & $4.919^{*} 10^{-1}$ \\
\hline
\end{tabular}

The relative gain in SNR achieved by OT as compared with FT is plotted in Fig. 8 using Eq. 10. The maximum achievable relative SNR gain is about $2.24 \mathrm{~dB}$ for $p=0.1$, $1.16 \mathrm{~dB}$ for $p=0.01$, and $1.73 \mathrm{~dB}$ for $p=0.03$. Figure 8 shows that the relative SNR gain is never less than 0 for all probabilities. This means that blanking nonlinearity with OT never results in lower SNR than blanking nonlinearity with FT. To further check the accuracy of the proposed method, the relative SNR gain was also plotted simulating a 64 QAM modulation. Figure 9 shows that the gain is always greater than 0 even with higher modulation index.

$$
S N R_{R G}(d B)=10 \log _{10}\left(S N R_{O T} / S N R_{F T}\right)
$$

System performance of blanking nonlinearity with OT is also compared with FT in terms of symbol-error rate (SER). Figures 10, 11, and 12 show SER for $p=0.01$, 0.03 , and 0.1 , respectively. Table 1 shows that the minimum SER achieved by OT is always lower than that achieved by the FT method in all IN scenarios. The SER of the system is given in Eq. 11 as in [6] as follows:

$$
\mathrm{SER}=1-\left[1-2\left(1-\frac{1}{\sqrt{m}}\right) Q\left(\sqrt{\frac{3 \gamma}{m-1}}\right)\right]^{2}
$$

Where $m$ is modulation index and $Q$ is $Q$ function.

\section{Conclusions}

The optimized threshold for blanking nonlinearity for achieving higher SNR and lower SER is proposed. As the optimized threshold is determined according to the peak, mean, and median of the signal, it gives better results and better system performance regardless of the probability of impulsive noise occurrence. On the other hand, a fixed threshold is independent of the signal characteristics. Therefore, one fixed threshold is not suitable for all the received signals with different probabilities of impulsive noise which may occur in practical scenarios.

Moreover, in this paper, the optimum range for setting $\gamma$ is introduced. Changing $\gamma$ within the given range does not cause a greater change in SNR. The SNR remains higher than that achieved by setting a fixed threshold. Whereas, changing the conventional fixed threshold results in a drastic change in SNR.

\section{Competing interests}

This work has been financially supported by the project fund provided by Wired and Wireless Communications Systems Laboratory, Gwangju Institute of Science and Technology, Gwangju, South Korea.

\section{Acknowledgements}

The authors are extremely thankful for the support and facilities provided by Gwangju Institute of Science and Technology, Gwangju, South Korea under the supervision of Professor Chang Soo Park.

Received: 23 December 2014 Accepted: 18 June 2015

Published online: 14 July 2015

\section{References}

1. TH Tran, DD Do, TH Huynh, PLC impulsive noise in industrial zone: measurement and characterization. Int J Comput Electrical Eng 5(1), 48-51 (2013)

2. J Lin, M Nassar, BL Evans, Impulsive noise mitigation in powerline communications using sparse Bayesian learning. IEEE Commun J 31(7), 1172-1183 (2013)

3. L Lampe, Bursty impulse noise detection by compressed sensing, in IEEE International Symposium on Power Line Communications and Its Applications (ISPLC), 2011, pp. 29-34

4. M Ghosh, Analysis of the effect of impulse noise on multicarrier and single carrier QAM systems. IEEE Trans Commun 44(2), 145-147 (1996)

5. SV Zhidkov, On the analysis of OFDM receiver with blanking nonlinearity in impulsive noise channels, in Proceedings of International Symposium on Intelligent Signal Processing and Communication Systems (ISPACS), 2004, pp. 492-496

6. SV Zhidkov, Performance analysis and optimization of OFDM receiver with blanking nonlinearity in impulsive noise environment. IEEE Trans Vehicular Technol 55(1), 234-242 (2006)

7. U Epple, M Schnell, Adaptive threshold optimization for a blanking nonlinearity in OFDM receivers, in IEEE Global Communications Conference (GLOBECOM), 2012, pp. 3661-3666

8. E Alsusa, KM Rabie, Dynamic peak-based threshold estimation method for mitigating impulsive noise in power-line communication systems. IEEE Trans Power Deliv 28(4), 2201-2208 (2013)

9. S Singhal, W Thakare, Comparative evaluation of standard and adaptive median filter for removing different type of noises. Int J Emerg Trends Sci Technol 1(07), 1227-1232 (2014)

10. T Sunilkumar, A Srinivas, ME Reddy, DGR Reddy, Removal of high density impulse noise through modified non-linear filter. J Theor Appl Inf Technol 47(2), 471-478 (2013)

\section{Submit your manuscript to a SpringerOpen ${ }^{\circ}$ journal and benefit from: \\ - Convenient online submission \\ Rigorous peer review \\ - Immediate publication on acceptance \\ - Open access: articles freely available online \\ - High visibility within the field \\ - Retaining the copyright to your article}

Submit your next manuscript at $>$ springeropen.com 